\title{
The Exact Frequency Equations for the Rayleigh and Shear Beams with Boundary Damping
}

\author{
Matthew P. Coleman and Laura A. McSweeney \\ Fairfield University, Department of Mathematics, Fairfield, CT 06824.
}

(Received 15 August 2017; accepted 26 January 2018)

While the Euler-Bernoulli beam is the most commonly utilized model in studying vibrating beams, one often requires a model that captures the additional effects of rotary inertia or deformation due to shear. The Rayleigh beam improves upon the Euler-Bernoulli by including the former effect, while the shear beam is an improvement that includes the latter. While all of these problems have been well studied when subject to energy-conserving boundary conditions, none have been solved for the case of boundary damping. We compute the exact frequency equations for the Rayleigh and shear beams, subject to boundary damping and, in the process, we find interesting connections between the two models, despite their being very different.

While the Euler-Bernoulli beam is the most commonly utilized model in studying vibrating beams, one often requires a model that captures the additional effects of rotary inertia or deformation due to shear. The Rayleigh beam improves upon the Euler-Bernoulli by including the former effect, while the shear beam is an improvement that includes the latter. While all of these problems have been well studied when subject to energy-conserving boundary conditions, none have been solved for the case of boundary damping. We compute the exact frequency equations for the Rayleigh and shear beams, subject to boundary damping and, in the process, we find interesting connections between the two models, despite their being very different.

\section{INTRODUCTION}

The two most commonly utilized linear models for studying the transverse vibration of beams are the Euler-Bernoulli and Timoshenko beams. The Euler-Bernoulli is, by far, the simpler of the two, including only the kinetic energy due to lateral displacement and the strain energy due to bending. The Timoshenko beam improves upon the Euler-Bernoulli by including the effects of rotary inertia and deformation due to shear. (There are many, many references for both models in the literature. See Han et al. ${ }^{1}$ and Traill-Nash and Collar ${ }^{2}$ for detailed descriptions and comparisons, for the case of energyconserving boundary conditions.) As such, the Timoshenko model is more complex and unwieldy than the Euler-Bernoulli. It is fortunate that, in so many engineering applications, the aspect ratio (i.e., the ratio of length to vertical thickness) usually is much greater than 10 , in which case the shear effects are negligible and the Euler-Bernoulli model is more appropriate. ${ }^{3,4}$

In some cases, though, the Euler-Bernoulli model may not be sufficient for a given situation, and one may need to consider, in addition, the effect of rotary inertia or of shear deformation, but not both. Thus, there are two standard intermediate beam models, the Rayleigh and shear beams. ${ }^{1}$
The Rayleigh beam is the improvement on the EulerBernoulli model that, additionally, considers the effect of rotary inertia. ${ }^{1,5}$ The shear beam, on the other hand, improves on the Euler-Bernoulli by including the effect of shear deformation. ${ }^{1,2}$ (We note that there are two other "shear" models - the simple shear beam, which includes only the lateral displacement and shear deformation; and the pure shear beam, which includes only the shear deformation and rotary inertia. ${ }^{1,6}$ As such, neither of these is a direct improvement on the EulerBernoulli model. We also note that the shear beam treated herein does not include the effect of warping due to shear.)

For each of these four models — Euler-Bernoulli, Rayleigh, shear and Timoshenko - the exact equations for the wave numbers for the homogeneous problem, subject to all combinations of energy-conserving boundary conditions, have been derived, and these results have been gathered together in the excellent study by Han et al. ${ }^{1}$ Indeed, this seems to be the only reference to include the roller-supported (or sliding) boundary condition. As for problems subject to energy-dissipative boundary conditions, however, the only solutions in the literature are asymptotic approximations for the Euler-Bernoulli and Timoshenko. ${ }^{7,8}$

Here, then, we present the exact frequency equations for the Rayleigh and shear beams, subject to boundary damping, and compare the results with each other, and with the corresponding energy- conserving problems. We choose to treat these models together here because they are of similar complexity and, moreover, because of the surprising and compelling similarity of the results, given the obvious differences between the two models. This paper will be useful for those interested in studying the damped Rayleigh and shear beam problems numerically, and as a springboard to the corresponding Timoshenko beam problem.

The paper, then, is organized as follows. In Section 2, we use a standard energy method to derive the partial differential equations for the Rayleigh and shear beams, along with 
the associated energy-dissipative boundary conditions, and we present diagrams of passive damping mechanisms that physically realize these boundary conditions. In Section 3, we solve the Rayleigh problem, i.e., we derive the exact equation for the wave numbers. In Section 4, we do the same for the shear problem. Finally, in Section 5, we relate the dissipative results to the corresponding energy-conserving results, and then provide a comparison between the Rayleigh and shear results.

\section{THE PROBLEMS}

We consider a uniform beam of length $L$ and let $W(x, t)$ be the vertical deflection, and $\Phi(x, t)$ the bending angle, of the beam at point $x$ and time $t$. In addition, we have the following constant physical parameters: $A$, the cross-sectional area; $\rho$, the mass per unit volume; $E$, Young's modulus; $I$, the area moment of inertia; $k^{\prime}$, the shape factor; and $G$, the shear modulus. (Note that $k^{\prime}$ and $G$ are not relevant to the Rayleigh problem.)

The energy for the Rayleigh beam at any time $t$ is:

$$
\begin{aligned}
& E(t)= \\
& \frac{1}{2} \int_{0}^{L}\left[\rho A W_{t}^{2}(x, t)+E I W_{x x}^{2}(x, t)+\rho I W_{x t}^{2}(x, t)\right] d x ;
\end{aligned}
$$

(see Han et al. ${ }^{1}$ ). Here, we have used the fact that the bending angle $\Phi(x, t)=W_{x}(x, t)$ for the Rayleigh beam. Now, in order to ensure that energy is dissipated, we require that:

$$
\begin{aligned}
& 0>E^{\prime}(t)= \\
& \begin{aligned}
\int_{0}^{L}\left[\rho A W_{t}(x, t) W_{t t}(x, t)+E I W_{x x}(x, t) W_{x x t}(x, t)\right. \\
\\
\left.\quad+\rho I W_{x t}(x, t) W_{x t t}(x, t)\right] d x .
\end{aligned}
\end{aligned}
$$

Integrating by parts, we have:

$$
\begin{gathered}
0>E^{\prime}(t)=E I W_{x x}(L, t) W_{x t}(L, t) \\
-E I W_{x x}(0, t) W_{x t}(0, t) \\
+\left[\rho I W_{x t t}(L, t)-E I W_{x x x}(L, t)\right] W_{t}(L, t) \\
-\left[\rho I W_{x t t}(0, t)-E I W_{x x x}(0, t)\right] W_{t}(0, t) \\
+\int_{0}^{L} W_{t}(x, t)\left[\rho A W_{t t}(x, t)\right. \\
\left.+E I W_{x x x x}(x, t)-\rho I W_{x x t t}(x, t)\right] d x .
\end{gathered}
$$

Requiring the integral to be zero yields the Rayleigh beam equation:

$$
\begin{array}{r}
\rho A W_{t t}(x, t)+E I W_{x x x x}(x, t)-\rho I W_{x x t t}(x, t)=0 ; \\
0<x<L ; t>0 .
\end{array}
$$

Then, we are ensured that $E^{\prime}(t)<0$ by choosing the following boundary conditions:

$$
\begin{aligned}
& \rho I W_{x t t}(0, t)-E I W_{x x x}(0, t)=\alpha_{1} W_{t}(0, t) ; \\
& E I W_{x x}(0, t)=\beta_{1} W_{x t}(0, t) ; \\
& \rho I W_{x t t}(L, t)-E I W_{x x x}(L, t)=-\alpha_{2} W_{t}(L, t) ; \\
& E I W_{x x}(L, t)=-\beta_{2} W_{x t}(L, t) ; t>0 ;
\end{aligned}
$$

where each damping constant $\alpha_{1}, \beta_{1}, \alpha_{2}, \beta_{2}$ is nonnegative and $\alpha_{1}+\beta_{1}+\alpha_{2}+\beta_{2}>0$. We have chosen to write Eqs. (5)(8) in the given form in order to reflect the facts that the bending angle $\Phi(x, t)$, the bending moment $M(x, t)$ and the shear force $Q(x, t)$ for the Rayleigh beam are:

$$
\begin{aligned}
& \Phi(x, t)=W_{x}(x, t) ; \\
& M(x, t)=E I W_{x x}(x, t) ; \\
& Q(x, t)=\rho I W_{x t t}(x, t)-E I W_{x x x}(x, t) ;
\end{aligned}
$$

(see the Appendix).

As for the shear beam, the energy is:

$$
\begin{aligned}
E(t)=\frac{1}{2} \int_{0}^{L}\{ & \rho A W_{t}^{2}(x, t)+E I \Phi_{x}^{2}(x, t) \\
& \left.+k^{\prime} G A\left[W_{x}(x, t)-\Phi(x, t)\right]^{2}\right\} d x ;
\end{aligned}
$$

(again, see Han et al. ${ }^{1}$ ) and, after a derivation similar to the one above, we arrive at the shear beam equations:

$$
\begin{aligned}
& \rho A W_{t t}(x, t)+k^{\prime} G A\left[\Phi_{x}(x, t)-W_{x x}(x, t)\right]=0 \\
& E I \Phi_{x x}(x, t)+k^{\prime} G A\left[W_{x}(x, t)-\Phi(x, t)\right]=0 \\
& 0<x<L ; \quad t>0
\end{aligned}
$$

which can be decoupled into the PDEs:

$$
\begin{aligned}
& \rho A W_{t t}(x, t)+E I W_{x x x x}(x, t)-\frac{\rho}{k^{\prime} G} W_{x x t t}(x, t)=0 \\
& \rho A \Phi_{t t}(x, t)+E I \Phi_{x x x x}(x, t)-\frac{\rho}{k^{\prime} G} \Phi_{x x t t}(x, t)=0 \\
& 0<x<L ; \quad t>0
\end{aligned}
$$

In addition, we have the dissipative boundary conditions:

$$
\begin{aligned}
& k^{\prime} G A\left[W_{x}(0, t)-\Phi(0, t)\right]=\alpha_{1} W_{t}(0, t) ; \\
& E I \Phi_{x}(0, t)=\beta_{1} \Phi_{t}(0, t) ; \\
& k^{\prime} G A\left[W_{x}(L, t)-\Phi(L, t)\right]=-\alpha_{2} W_{t}(L, t) ; \\
& E I \Phi_{x}(L, t)=-\beta_{2} \Phi_{t}(L, t) ; t>0 ;
\end{aligned}
$$

where the damping constants are as above. We note that, for the shear beam, the bending angle, bending moment and shear are given, respectively, by:

$$
\begin{aligned}
& \Phi(x, t)=\frac{E I}{k^{\prime} G A} W_{x x x}(x, t) \\
& +W_{x}(x, t)-\frac{\rho E I}{\left(k^{\prime} G\right)^{2} A} W_{x t t}(x, t) ; \\
& M(x, t)=E I \Phi_{x}(x, t) ; \\
& Q(x, t)=k^{\prime} G A\left[W_{x}(x, t)-\Phi(x, t)\right] ;
\end{aligned}
$$

(again, see the Appendix).

Following the literature, we perform the standard separation of variables:

$$
W(x, t)=\mathrm{e}^{-\mathrm{i} \xi^{2} t} w(x) ; \Phi(x, t)=\mathrm{e}^{-\mathrm{i} \xi^{2} t} \phi(x) ;
$$


and then, following Coleman and Schaffer', we introduce the dimensionless quantities:

$$
\begin{aligned}
y=\frac{x}{L} ; w=\frac{v}{L} ; \quad k^{2}=\sqrt{\frac{\rho A}{E I}} \frac{1}{L^{2}} \xi^{2} ; \\
\gamma_{1}=\frac{I}{A L^{2}} ; \gamma_{2}=\frac{E I}{k^{\prime} G A L^{2}} ;
\end{aligned}
$$

and abuse notation by letting $y \rightarrow x$ and $v \rightarrow w$, and the Rayleigh PDE in Eq. (4) leads to the ODE:

$$
w^{(4)}(x)+\gamma_{1} k^{4} w^{\prime \prime}(x)-k^{4} w(x)=0 ; \quad 0<x<1 ;
$$

while the shear PDEs in Eqs. (13)-(16) result in the ODEs:

$$
\begin{aligned}
& \phi^{\prime}(x)-w^{\prime \prime}(x)-\gamma_{2} k^{4} w(x)=0 \\
& w^{\prime}(x)-\phi(x)+\gamma_{2} \phi^{\prime \prime}(x)=0 \\
& w^{(4)}(x)+\gamma_{2} k^{4} w^{\prime \prime}(x)-k^{4} w(x)=0 \\
& \phi^{(4)}(x)+\gamma_{2} k^{4} \phi^{\prime \prime}(x)-k^{4} \phi(x)=0 .
\end{aligned}
$$

As for the boundary conditions, we first introduce the dimensionless quantities:

$$
a_{j}=\frac{L}{\sqrt{\rho A E I}} \alpha_{j} ; \quad b j=\frac{1}{L \sqrt{\rho A E I}} \beta_{j} ; j=1,2 .
$$

Then, the separated dimensionless versions of Eqs. (5)-(8) become:

$$
\begin{aligned}
& w^{\prime \prime \prime}(0)+\gamma_{1} k^{4} w^{\prime}(0)-\mathrm{i} a_{1} k^{2} w(0)=0 \\
& w^{\prime \prime}(0)+\mathrm{i} b_{1} k^{2} w^{\prime}(0)=0 ; \\
& w^{\prime \prime \prime}(1)+\gamma_{1} k^{4} w^{\prime}(1)+\mathrm{i} a_{2} k^{2} w(1)=0 \\
& w^{\prime \prime}(1)-\mathrm{i} b_{2} k^{2} w^{\prime}(1)=0 ;
\end{aligned}
$$

while Eqs. (17)-(20) become:

$$
\begin{aligned}
& w^{\prime}(0)-\phi(0)+\mathrm{i} a_{1} k^{2} w(0)=0 \\
& \phi^{\prime}(0)+\mathrm{i} b_{1} k^{2} \phi(0)=0 \\
& w^{\prime}(1)-\phi(1)-\mathrm{i} a_{2} k^{2} w(1)=0 \\
& \phi^{\prime}(1)-\mathrm{i} b_{2} k^{2} \phi(1)=0 .
\end{aligned}
$$

Before solving these problems, we present here, in Figs. 1 and 2, passive damping mechanisms for realizing the dissipative boundary conditions. Specifically, Fig. 1 corresponds to the boundary conditions Eqs. (7) and (19), while Fig. 2 illustrates Eqs. (8) and (20).

In Fig. 1, we have a vertical linear damper, with damping coefficient $c_{V}$, at $x=1$. As the velocity is $W_{t}(1, t)$, the resulting shear force is:

$$
Q(1, t)=-c_{V} W_{t}(1, t) .
$$

In Fig. 2, we have a horizontal linear damper, with damping coefficient $c_{H}$, at $\mathrm{x}=1$, at a distance $\frac{h}{2}$ from the beam's neutral axis. Here, $h$ is the beam's vertical thickness. As the horizontal velocity at the damper is $\Phi(1, t) \frac{h}{2}$, the resulting bending moment is:

$$
M(1, t)=c_{H} \frac{h}{2} \text { velocity }=c_{H} \Phi(1, t) \frac{h^{2}}{4} .
$$

(We note that the sign in Eq. (39) differs from that in Chen et al. ${ }^{7}$ This is due purely to the sign convention used for the bending moment.)

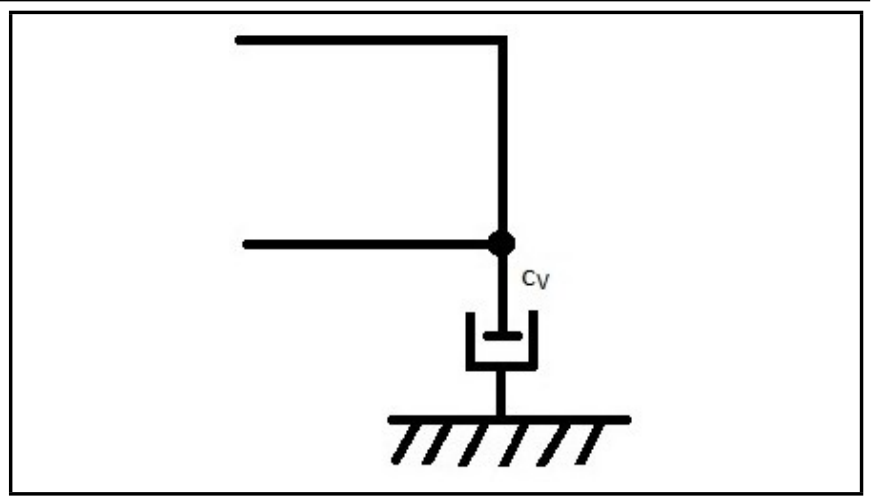

Figure 1. Vertical damper at right end $x=1$, for realizing boundary conditions in Eqs. (7) and (19).

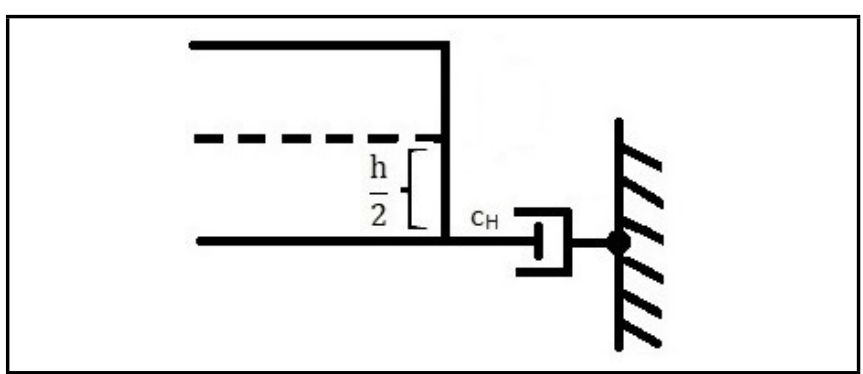

Figure 2. Horizontal damper at right end $x=1$, for realizing boundary conditions in Eqs. (8) and (20).

\section{SOLUTION OF THE RAYLEIGH BEAM PROBLEM}

The general solution of the Rayleigh ODE in Eq. (24) is:

$w(x)=c_{1} \cos a x+c_{2} \sin a x+c_{3} \cosh b x+c_{4} \sinh b x ;$

where $c_{j}, j=1, \ldots, 4$, are arbitrary constants and the dispersion relations for the wave numbers $a$ and $b$ are:

$$
\left\{\begin{array}{l}
a \\
b
\end{array}\right\}=k^{2} \sqrt{\sqrt{\left(\frac{\gamma_{1}}{2}\right)^{2}+\frac{1}{k^{4}}} \pm \frac{\gamma_{1}}{2}}
$$

We note that Eq. (41) implies that:

$$
k^{4}=a^{2} b^{2} ; \quad \gamma_{1} k^{4}=a^{2}-b^{2}
$$

(The above computations are equivalent to those in Han et al. ${ }^{1}$ )

We apply the boundary conditions in Eqs. (30)-(33) to Eq. (40) and, using Eq. (42), the frequency equation is:

$$
\left[\begin{array}{cccc}
-a^{2} & \mathrm{i} b_{1} a^{2} b & b^{2} & \mathrm{i} b_{1} a b^{2} \\
\mathrm{i} a_{1} & b & \mathrm{i} a_{1} & -a \\
z_{31} & z_{32} & z_{33} & z_{34} \\
z_{41} & z_{42} & z_{43} & z_{44}
\end{array}\right]=0
$$


where:

$$
\begin{aligned}
& z_{31}=\mathrm{i} b_{2} a^{2} b \sin a-a^{2} \cos a ; \\
& z_{32}=-\left(\mathrm{i} b_{2} a^{2} b \cos a+a^{2} \sin a\right) ; \\
& z_{33}=b^{2} \cosh b-\mathrm{i} b_{2} a b^{2} \sinh b ; \\
& z_{34}=b^{2} \sinh b-\mathrm{i} b_{2} a b^{2} \cosh b ; \\
& z_{41}=b \sin a+\mathrm{i} a_{2} \cos a ; \\
& z_{42}=-b \cos a+\mathrm{i} a_{2} \sin a ; \\
& z_{43}=a \sinh b+\mathrm{i} a_{2} \cosh b ; \\
& z_{44}=a \cosh b+\mathrm{i} a_{2} \sinh b .
\end{aligned}
$$

After very much, but straightforward, computation, the frequency equation becomes:

$$
\begin{aligned}
& -2 a^{3} b^{3}+2 a^{3} b^{3} \cos a \cosh b+\left(b^{6}-a^{6}\right) \sin a \sinh b \\
& +\left(a_{1} b_{1}+a_{2} b_{2}\right) a b\left[2 a^{2} b^{2}+\left(a^{4}+b^{4}\right) \cos a \cosh b\right. \\
& \left.+a b\left(b^{2}-a^{2}\right) \sin a \sinh b\right] \\
& +\left(a_{1} a_{2}-b_{1} b_{2} a^{2} b^{2}\right)\left(a^{2}+b^{2}\right)^{2} \sin a \sinh b \\
& +\left(a_{1} b_{2}+a_{2} b_{1}\right) a b\left(a^{2}+b^{2}\right)^{2} \cos a \cosh b \\
& +a_{1} b_{1} a_{2} b_{2} a^{2} b^{2}[-2 a b+2 a b \cos a \cosh b \\
& \left.+\left(a^{2}-b^{2}\right) \sin a \sinh b\right] \\
& +\mathrm{i}\left(a^{2}+b^{2}\right)\left\{\left(a_{1}+a_{2}\right)\left(b^{3} \cos a \sinh b-a^{3} \sin a \cosh b\right)\right. \\
& -\left(b_{1}+b_{2}\right) a b\left(a^{3} \cos a \sinh b+b^{3} \sin a \cosh b\right) \\
& +a_{1} a_{2}\left(b_{1}+b_{2}\right) a b(a \cos a \sinh b-b \sin a \cosh b) \\
& \left.-b_{1} b_{2}\left(a_{1}+a_{2}\right) a^{2} b^{2}(b \cos a \sinh b+a \sin a \cosh b)\right\}=0 .
\end{aligned}
$$

For comparison, we have also computed the frequency equation for the corresponding Euler-Bernoulli beam problem, and we have found that letting $b \rightarrow a$ in Eq. (45) (i.e., $\gamma_{1} \rightarrow 0$, which occurs when the aspect/slenderness ratio $\rightarrow \infty$ ) does, indeed, yield the Euler-Bernoulli equation. Note also that we should be able to recover from Eq. (45) the frequency equations for all of the conservative Rayleigh beam problems using the following:

$$
\begin{aligned}
& a_{j}=b_{j}=0 \Rightarrow \text { that end is free }(F): \\
& \begin{array}{l}
M=Q=0 \text { at that end; } \\
a_{j}=b_{j}=\infty \Rightarrow \text { that end is clamped }(C): \\
W=\Phi=0 ; \\
a_{j}=\infty, b_{j}=0 \Rightarrow \text { that end is simply supported }(S): \\
W=M=0 ; \\
a_{j}=0, b_{j}=\infty \Rightarrow \text { that end is roller-supported }(R): \\
\Phi=Q=0 .
\end{array}
\end{aligned}
$$

(Note that simply supported sometimes is referred to as hinged or pinned, while sliding sometimes is used instead of rollersupported.) There are ten such conservative problems and we provide them here:

$$
\begin{aligned}
& C-C: \quad-2 a b+2 a b \cos a \cosh b \\
& +\left(a^{2}-b^{2}\right) \sin a \sinh b=0 ; \\
& C-S: \quad a \cos a \sinh b-b \sin a \cosh b=0 ; \\
& C-R: \quad b \cos a \sinh b+a \sin a \cosh b=0 ; \\
& C-F: \quad 2 a^{2} b^{2}+\left(a^{4}+b^{4}\right) \cos a \cosh b \\
& +a b\left(b^{2}-a^{2}\right) \sin a \sinh b=0 ; \\
& S-S: \quad \sin a \sinh b=0 ; \\
& S-R: \quad \cos a \cosh b=0 ; \\
& S-F: \quad b^{3} \cos a \sinh b-a^{3} \sin a \cosh b=0 ; \\
& R-R: \quad \sin a \sinh b=0 ; \\
& R-F: \quad a^{3} \cos a \sinh b+b^{3} \sin a \cosh b=0 ; \\
& F-F: \quad-2 a^{3} b^{3}+2 a^{3} b^{3} \cos a \cosh b \\
& +\left(b^{6}-a^{6}\right) \sin a \sinh b=0 ;
\end{aligned}
$$

(where, e.g., $C-F$ means that one end is clamped and the other is free). It is easy to see that Eqs. (50)-(59) follow from applying the appropriate equations from Eq. (46)-(49) to Eq. (45). (Some of Eqs. (50)-(59) can be found in Han et al. ${ }^{1}$ )

\section{SOLUTION OF THE SHEAR BEAM PROBLEM}

The general solutions of the shear ODEs in Eqs. (27) and (28) are:

$$
w(x)=c_{1} \cos a x+c_{2} \sin a x+c_{3} \cosh b x+c_{4} \sinh b x ;
$$

and:

$$
\phi(x)=d_{1} \cos a x+d_{2} \sin a x+d_{3} \cosh b x+d_{4} \sinh b x ;
$$

where $c_{j}$ and $d_{j}, j=1, \ldots, 4$, are arbitrary constants and where, exactly as in the case of the Rayleigh beam, the dispersion relations for the wave numbers are:

$$
\left\{\begin{array}{l}
a \\
b
\end{array}\right\}=k^{2} \sqrt{\sqrt{\left(\frac{\gamma_{2}}{2}\right)^{2}+\frac{1}{k^{4}}} \pm \frac{\gamma_{2}}{2}}
$$

which, again, gives us:

$$
k^{4}=a^{2} b^{2} ; \quad \gamma_{2} k^{4}=a^{2}-b^{2} .
$$

In addition, the constants $c_{j}$ and $d_{j}$ are related via Eq. (25) which, when combined with Eq. (63), leads to

$$
d_{1}=\frac{b^{2}}{a} c_{2} ; \quad d_{2}=-\frac{b^{2}}{a} c_{1} ; \quad d_{3}=\frac{a^{2}}{b} c_{4} ; \quad d_{4}=\frac{a^{2}}{b} c_{3} .
$$

(Again, the above computations are equivalent to those in Han et al. $^{1}$ )

Finally, applying the boundary conditions in Eqs. (34)-(37) to Eqs. (60) and (61), and using Eqs. (63) and (64), we have the frequency equation:

$$
\left[\begin{array}{cccc}
\mathrm{i} a_{1} a^{2} b^{2} & b\left(a^{2}-b^{2}\right) & \mathrm{i} a_{1} a^{2} b^{2} & a\left(b^{2}-a^{2}\right) \\
-b^{2} & \mathrm{i} b_{1} b^{3} & a^{2} & \mathrm{i} b_{1} a^{3} \\
z_{31} & z_{32} & z_{33} & z_{34} \\
z_{41} & z_{42} & z_{43} & z_{44}
\end{array}\right]=0 ;
$$


where:

$$
\begin{aligned}
& z_{31}=b\left(b^{2}-a^{2}\right) \sin a-\mathrm{i} a_{2} a^{2} b^{2} \cos a ; \\
& z_{32}=b\left(a^{2}-b^{2}\right) \cos a-\mathrm{i} a_{2} a^{2} b^{2} \sin a ; \\
& z_{33}=a\left(b^{2}-a^{2}\right) \sinh b-\mathrm{i} a_{2} a^{2} b^{2} \cosh b ; \\
& z_{34}=a\left(b^{2}-a^{2}\right) \cosh b-\mathrm{i} a_{2} a^{2} b^{2} \sinh b ; \\
& z_{41}=b^{2}\left(-\cos a+\mathrm{i} b_{2} b \sin a\right) ; \\
& z_{42}=-b^{2}\left(-\sin a+\mathrm{i} b_{2} b \cos a\right) ; \\
& z_{43}=a^{2}\left(\cosh b-\mathrm{i} b_{2} a \sinh b\right) ; \\
& z_{44}=a^{2}\left(\sinh b-\mathrm{i} b_{2} a \cosh b\right) .
\end{aligned}
$$

Then, again, after much computation, the frequency equation becomes:

$$
\begin{aligned}
& a^{2} b^{2}\left[-2 a b+2 a b \cos a \cosh b+\left(a^{2}-b^{2}\right) \sin a \sinh b\right] \\
& +\left(a_{1} b_{1}+a_{2} b_{2}\right) a b\left[2 a^{2} b^{2}+\left(a^{4}+b^{4}\right) \cos a \cosh b\right. \\
& \left.+a b\left(b^{2}-a^{2}\right) \sin a \sinh b\right] \\
& +\left(a_{1} a_{2}-b_{1} b_{2} a^{2} b^{2}\right)\left(a^{2}+b^{2}\right) \sin a \sinh b \\
& +\left(a_{1} b_{2}+a_{2} b_{1}\right) a b\left(a^{2}+b^{2}\right)^{2} \cos a \cosh b \\
& +a_{1} b_{1} a_{2} b_{2}\left[-2 a^{3} b^{3}+2 a^{3} b^{3} \cos a \cosh b\right. \\
& \left.+\left(b^{6}-a^{6}\right) \sin a \sinh b\right] \\
& +\mathrm{i}\left(a^{2}+b^{2}\right)\left\{\left(a_{1}+a_{2}\right) a b(a \cos a \sinh b-b \sin a \cosh b)\right. \\
& -\left(b_{1}+b_{2}\right) a^{2} b^{2}(a \sin a \cosh b+b \cos a \sinh b) \\
& +a_{1} a_{2}\left(b_{1}+b_{2}\right)\left(b^{3} \cos a \sinh b-a^{3} \sin a \cosh b\right) \\
& \left.-b_{1} b_{2}\left(a_{1}+a_{2}\right) a b\left(a^{3} \cos a \sinh b+b^{3} \sin a \cosh b\right)\right\}=0 .
\end{aligned}
$$

Again, letting $b \rightarrow a$ in Eq. (67) (i.e., $\gamma_{2} \rightarrow 0$, as we let the shear modulus $G \rightarrow \infty$ ) results in the Euler-Bernoulli equation. In addition, using Eqs. (46)-(49) should allow us to recover the various conservative frequency equations, which we provide here:

$$
\begin{aligned}
& C-C: \quad-2 a^{3} b^{3}+2 a^{3} b^{3} \cos a \cosh b \\
& +\left(b^{6}-a^{6}\right) \sin a \sinh b=0 ; \\
& C-S: \quad b^{3} \cos a \sinh b-a^{3} \sin a \cosh b=0 ; \\
& C-R: \quad a^{3} \cos a \sinh b+b^{3} \sin a \cosh b=0 ; \\
& C-F: \quad 2 a^{2} b^{2}+\left(a^{4}+b^{4}\right) \cos a \cosh b \\
& +a b\left(b^{2}-a^{2}\right) \sin a \sinh b=0 ; \\
& S-S: \quad \sin a \sinh b=0 ; \\
& S-R: \quad \cos a \cosh b=0 ; \\
& S-F: \quad a \cos a \sinh b-b \sin a \cosh b=0 ; \\
& R-R: \quad \sin a \sinh b=0 ; \\
& R-F: \quad a \sin a \cosh b+b \cos a \sinh b=0 ; \\
& F-F: \quad-2 a b+2 a b \cos a \cosh b \\
& +\left(a^{2}-b^{2}\right) \sin a \sinh b=0 .
\end{aligned}
$$

It is easy to see that Eqs. (68)-(77) follow from applying the appropriate equations from Eqs. (46)-(49) to Eq. (67). (And again, some of Eqs. (68)-(77) are given in Han et al. $\left.{ }^{1}\right)$

\section{A BRIEF COMPARISON OF THE RESULTS}

Although the Rayleigh and shear PDEs, and separated ODEs, are identical in form, the expressions for the bending angle, bending moment and shear force are very different for the two beams. Thus, it is very surprising to see the similarities between Eqs. (45) and (67). Indeed, every term in Eq. (45) corresponds exactly to a term in Eq. (67), except that the damping constant coefficients are different in some cases. It follows, of course, that every equation in Eqs. (50)-(59) has an exact counterpart in Eqs. (68)-(77), except that the corresponding boundary conditions differ in some cases. (This last fact is referred to briefly in Han et al. ${ }^{1}$ ) In fact, it is easy to see that we can get from Eqs. (50)-(59) to Eqs. (68)-(77) simply by interchanging $C$ and $F$. Unfortunately, we have been unable to find a physical interpretation of this phenomenon, nor can we suggest one ourselves.

It is easy to extend this idea to the dissipative equations Eqs. (45) and (67). Consider, e.g., the term in Eq. (45) containing the product $a_{1} b_{1} a_{2}$. When letting $a_{1}=a_{2}=b_{1}=$ $\infty, b_{2}=0$, this gives us the $C-S$ equation Eq. (51). Let us, then, refer to this as a $C-S$ term of Eq. (45) (there will be another, corresponding to $a_{1} a_{2} b_{2}$ ). Now the same term appears in Eq. (67), but with only $a_{2}$ as its coefficient, so it is an $S-F$ term of Eq. (67) (again, there is another, corrresponding to $\left.a_{1}\right)$. Thus, we can use the interchange of $C$ with $F$ in order to get from Eq. (45) to Eq. (67), and vice versa. This is equivalent to the following: if a term includes the product $a_{j} b_{j}$, remove it; if a term includes neither $a_{j}$ nor $b_{j}$, multiply it by $a_{j} b_{j}$. In Table 1 we give the correspondence between the terms in Eqs. (45) and (67).

Again, as with the conservative problems, we cannot suggest a physical interpretation of this phenomenon, at present. We hope that future researchers will shed some light on this question.

\section{REFERENCES}

1 Han, S. M., Benaroya, H., and Wei, T. Dynamics of transversely vibrating beams using four engineering theories. Journal of Sound and Vibration, 225(5), 935-988, 1999. https://dx.doi.org/10.1006/jsvi.1999.2257.

2 Traill-Nash, R. W. and Collar, A. R. The effects of shear flexibility and rotatory inertia on the bending vibrations of beams. Quarterly Journal of Mechanics and Applied Mathematics, 6, 186-222, 1953. https://dx.doi.org/10.1093/qjmam/6.2.186.

3 Simão, P. D. Influence of shear deformations on the buckling of columns using the Generalized Beam Theory and energy principles. European Journal of Mechanics A/Solids, 61, 216-234, 2017. https://dx.doi.org/10.1016/j.euromechsol.2016.09.015. 
Table 1. Correspondence between terms in damped Rayleigh and damped shear beam frequency equations.

\begin{tabular}{||c|c|c|c||}
\hline \multicolumn{2}{|c|}{ Rayleigh } & \multicolumn{2}{c|}{ Shear } \\
\hline Term & Damping Coefficients & Term & Damping Coefficients \\
\hline$C-C$ & $a_{1} b_{1} a_{2} b_{2}$ & $F-F$ & - \\
\hline$C-S, S-C$ & $a_{1} b_{1} a_{2}, a_{1} a_{2} b_{2}$ & $F-S, S-F$ & $a_{2}, a_{1}$ \\
\hline$C-R, R-C$ & $a_{1} b_{1} b_{2}, b_{1} a_{2} b_{2}$ & $F-R, R-F$ & $b_{2}, b_{1}$ \\
\hline$C-F, F-C$ & $a_{1} b_{1}, a_{2} b_{2}$ & $F-C, C-F$ & $a_{2} b_{2}, a_{1} b_{1}$ \\
\hline$S-S$ & $a_{1} a_{2}$ & $S-S$ & $a_{1} a_{2}$ \\
\hline$S-R, R-S$ & $a_{1} b_{2}, a_{2} b_{1}$ & $S-R$ & $a_{1} b_{2}, a_{2} b_{1}$ \\
\hline$S-F, F-S$ & $a_{1}, a_{2}$ & $S-C, C-S$ & $a_{1} a_{2} b_{2}, a_{1} b_{1} a_{2}$ \\
\hline$R-R$ & $b_{1} b_{2}$ & $R-R$ & $b_{1} b_{2}$ \\
\hline$R-F, F-R$ & $b_{1}, b_{2}$ & $C-R, R-C$ & $b_{1} a_{2} b_{2}, a_{1} b_{1} a_{2}$ \\
\hline$F-F$ & - & $C-C$ & $a_{1} b_{1} a_{2} b_{2}$ \\
\hline
\end{tabular}

4 Chopra, A. K. Dynamics of Structure - Theory and Applications to Earthquake Engineering. Prentice Hall, 2001.

5 Strutt, J. W. Theory of Sound. Macmillan, 1877. https://dx.doi.org/10.1017/CBO9781139058087.

6 Abbas, B. A. B. and Thomas, J. The second frequency spectrum of Timoshenko beams. Journal of Sound and Vibration, 51, 123-137, 1977. https://dx.doi.org/10.1016/s0022460x(77)80118-1.

7 Chen, G., Krantz, S. G., Ma, D. W., Wayne, C. E., and West, H. H. The Euler-Bernoulli beam equation with boundary energy dissipation. In S. Lee, editor, Operator Methods for Optimal Control Problems, 67-96. Marcel-Dekker, 1987. https://dx.doi.org/10.21236/ada189517.

8 Coleman, M. P. and Wang, H. K. Analysis of the vibration spectrum of a Timoshenko Beam with boundary damping by the wave method. Wave Motion, 17, 223-239, 1993. https://dx.doi.org/10.1016/0165-2125(93)90003-x.

9 Coleman, M. P. and Schaffer, L. Asymptotic analysis of the vibration spectrum of coupled Timoshenko beams with a dissipative joint. European Journal of Mechanics A/Solids, 29(4), 629-636, 2010. https://dx.doi.org/10.1016/j.euromechsol.2010.03.004.

\section{APPENDIX A. DERIVATION OF EXPRESSION FOR $\Phi(X, T), M(X, T)$ AND $Q(X, T)$ IN TERMS OF $W(X, T)$}

For a Timoshenko beam, we have the relations: ${ }^{9}$

$$
\begin{aligned}
& Q_{x}(x, t)-\rho A W_{t t}(x, t)=0 \\
& Q(x, t)+M_{x}(x, t)-\rho I \Phi_{t t}(x, t)=0 ; \\
& W_{x}(x, t)-\Phi(x, t)=\frac{1}{k^{\prime} G A} Q(x, t) \\
& \Phi_{x}(x, t)=\frac{1}{E I} M(x, t) .
\end{aligned}
$$

The Rayleigh beam satisfies Eqs. (A.78), (A.79) and (A.81) but neglects the effect of shear deformation; thus, Eq. (A.80) must be replaced by:

$$
W_{x}(x, t)-\Phi(x, t)=0 .
$$

Then, Eqs. (A.79), (A.81) and (A.82) give us:

$$
\begin{aligned}
& \Phi(x, t)=W_{x}(x, t) ; \\
& M(x, t)=E I W_{x x}(x, t) ; \\
& Q(x, t)=\rho I W_{x t t}(x, t)-E I W_{x x x}(x, t) ;
\end{aligned}
$$

for a Rayleigh beam.

The shear beam satisfies Eqs. (A.78), (A.80) and (A.81), but neglects the rotary inertia term in Eq. (A.79); thus, Eq. (A.79) must be replaced by

$$
Q(x, t)+M_{x}(x, t)=0 .
$$

From Eqs. (A.78) and (A.80) we have the PDE in Eq. (13):

$$
\rho A W_{t t}(x, t)+k^{\prime} G A\left[\Phi_{x}(x, t)-W_{x x}(x, t)\right]=0 ;
$$

and solving for $\Phi$ gives us:

$$
\Phi(x, t)=W_{x}(x, t)-\frac{\rho}{k^{\prime} G} \int W_{t t}(x, t) d x .
$$

Then, as Eqs. (A.81) and (A.86) give us $M$ and $Q$ in terms of $\Phi$, we have:

$$
M(x, t)=E I W_{x x}(x, t)-\frac{\rho E I}{k^{\prime} G} W_{t t}(x, t)
$$

and:

$$
Q(x, t)=\frac{\rho E I}{k^{\prime} G} W_{x t t}(x, t)-E I W_{x x x}(x, t) .
$$

Lastly, from Eq. (A.80) we have:

$\Phi(x, t)=\frac{E I}{k^{\prime} G A} W_{x x x}(x, t)+W_{x}(x, t)-\frac{\rho E I}{\left(k^{\prime} G\right)^{2} A} W_{x t t}(x, t)$.

(Note that, indeed, we could have used Eqs. (A.78), (A.79), (A.81) and (A.82) to derive the Rayleigh PDE in Eq. (4), and Eqs. (A.78), (A.80), (A.81) and (A.86) to derive the shear PDEs in Eqs. (13) and (14). However, there is no natural way to derive from these the dissipative conditions.) 